\title{
Numerical simulation of S-shaped inlet under the intake total pressure distortion
}

\author{
Ying Liu, Xiaobo Zhang ${ }^{*}$, Yang Yu, Bingkun Yan, Congrui Cai, and Bateng Wu \\ Aeronautics Engineering College, Air Force Engineering University, Xi'an, 710038, China
}

\begin{abstract}
During the development of the stealth fighter, the S-shaped inlet enters the designer's vision because it has better stealth than bump inlet and straight inlet. During the use of the S-shaped inlet, due to its structural reasons, secondary flow is likely to occur in the curved section, which directly causes the flow state to be changeable and complicated. Therefore, this paper takes the S-shaped inlet as the research object to analyzes the steady flow field simulation under uniform inlet condition and distortion inlet condition and analyze the flow field of the airflow and the total pressure of each section under the S-shaped inlet by changing the intake distortion conditions with CFX software. The results show that although the S-shaped inlet will occur total pressure distortion under uniform intake. However, when the S-shaped inlet work under certain flight conditions, the level of total pressure distortion will be smaller than the uniform inlet condition, which can improve the air intake performance. Finally, it can be inferred that with use of the S-shaped intake port, the deterioration of distortion may be prevented under certain specific intake conditions.
\end{abstract}

Keywords: S-shaped Inlet, Intake total pressure distortion, Distortion index, Numerical simulation.

\section{Introduction}

In the development of stealth fighters, in order to pursue a better stealth effect, the Sshaped inlet has entered people's field of vision because it can reduce the RCS. However, the use of S-shaped inlet will affect the intake efficiency of the engine, so international experts and scholars have carried out experiments and analyses on its performance. $\mathrm{Xu}{ }^{[1]}$ et al. used a five-hole probe-based method for measuring and evaluating the total pressure distortion of the large S-shaped inlet, and conducted a more accurate evaluation and analysis of the total pressure distortion. Zhang ${ }^{[2]}$ used micro blades as vortex generators in low-speed wind tunnels to control the flow of an S-shaped inlet, and obtained a feasible method for controlling the total pressure distortion of the S-shaped inlet. In view of the high-test cost and long cycle, simulation can effectively reduce the workload and investment cost of engine product development and shorten the development cycle. Recent years, simulation studies and numerical calculations on the flow characteristics of the S-shaped inlet have been carried out ${ }^{[3-5]}$. Some researchers have carried out research and analysis with the focus on the total

\footnotetext{
*Corresponding author: xazxb@163.com
} 
pressure loss ${ }^{[6]}$. Guo ${ }^{[7]}$ and his co-workers have focused on the S-shaped inlet. Related experiments have been carried out on the flow law of the secondary stream. At the same time, for different turbulence models Xiao, Saha, Gerolymos, Zhang, ${ }^{[8-11]}$ were analysed the Sshaped the flow characteristics of the S-shaped inlet. Lin ${ }^{[12,13]}$ had also carried out related research on the S-shaped inlet at high angles of attack and the rectangular variable circular cross-section S-shaped inlet. In order to more comprehensively analyse the air flow status under S-shaped inlet at low speeds and large angles of attack. Weng ${ }^{[15]}$ conducted experiments with an S-shaped inlet under the belly as the research object.

Therefore, based on the historical research results, this paper explores the intake performance of the S-shaped inlet by simulating the flow field under the actual distortion conditions such as the angle of attack state and the suction ground vortex state, and comparing the effect of distortion index under the axial uniform intake distortion.

\section{Basic theory and mode}

\subsection{Distortion index}

In order to express the severity of the distorted flow field and measure the magnitude of the distortion, the distortion index is generally used to quantitatively measure the degree of distortion of the distorted flow field compared to the uniform flow field. This article chooses the steady-state circumferential total pressure unevenness $\Delta \bar{\sigma}$ to describe the characteristics of the total pressure distortion flow field. Equation1 gives the calculation formula of $\Delta \bar{\sigma}$.

$$
\Delta \bar{\sigma}=1-\frac{p_{a v l o w}^{*}}{p_{a v}^{*}}
$$

where $P_{a v l o w}^{*}$ is the average total pressure of low pressure region; $P_{a v}^{*}$ is the average total pressure value of the circumferential surface on the interface. Equation 2 gives the expression of $P_{\text {avlow }}^{*}$

$$
p_{\text {avlow }}^{*}=\frac{1}{\theta_{2}-\theta_{1}} \int_{\theta_{1}}^{\theta_{2}} p^{*}(\theta) d \theta
$$

where $\theta_{1} 、 \theta_{2}$ are the starting angle and ending angle of low pressure region. Equation 3 gives the expression of $P_{a v}^{*}$

$$
\begin{gathered}
P_{a v}^{*}=p_{i}^{*}+\frac{M}{2 \pi}\left(p_{i+1}^{*}-p_{i}^{*}\right)\left(\theta-\frac{2 \pi}{M} i\right) \\
\frac{2 \pi}{M} i \leqq \frac{2 \pi}{M}(i+1) \\
i=0 \sim M-1
\end{gathered}
$$

Figure.1 shows the mode of S-shaped inlet. It can be found that there is a significant gradient at the inflection point of the intake port, which will affect the intake performance. 


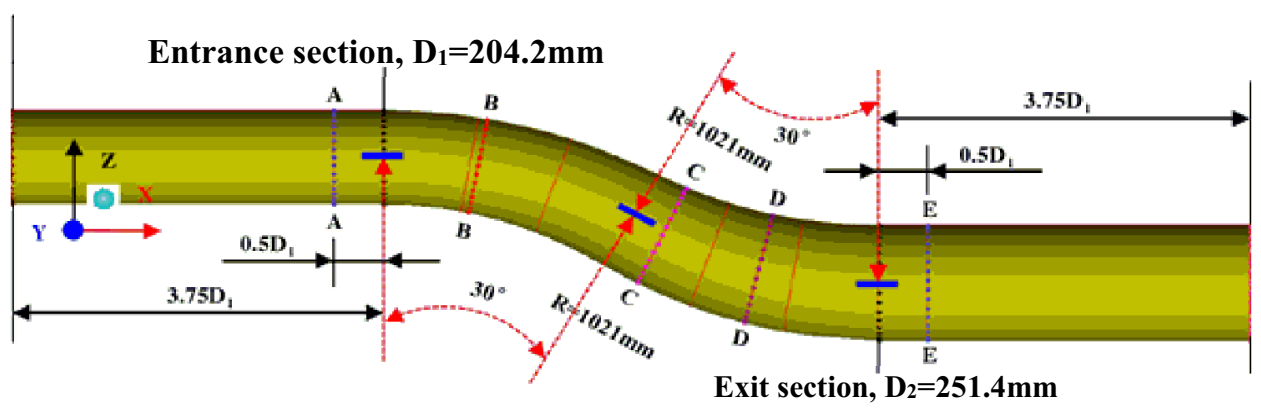

Fig. 1. S-shaped inlet mode.

\section{Results and analysis}

\subsection{Inlet distortion under uniform intake conditions}

Due to the special structure of the S-shaped inlet, under normal and uniform inlet conditions, the internal flow field will occur total pressure distortion. The phenomenon of air flow separation has an important impact on subsequent flow field changes. After calculation, the outlet distortion index under uniform intake conditions is $7.5 \%$. Figure. 2 shows the total pressure distribution under uniform intake conditions.

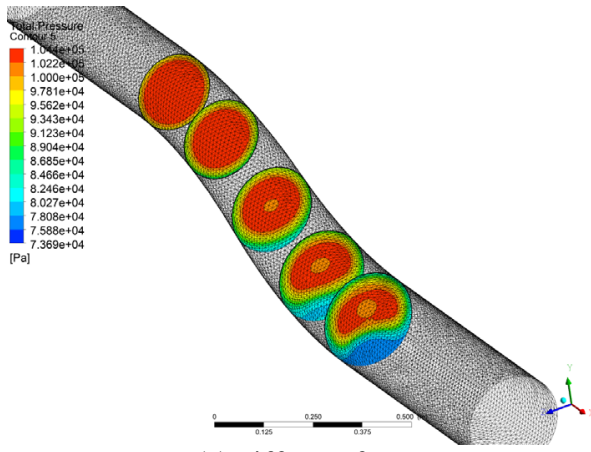

(a)Different faces

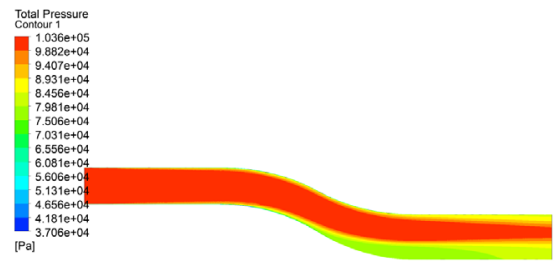

(b) Axial direction

Fig. 2. Total pressure distribution under uniform intake condition.

\subsection{Inlet distortion under angle of attack}

This section simulates the air intake distortion state of the S-shaped inlet under the flying condition of the elevation angle when the aircraft is manoeuvring at low speed. The total inlet pressure distribution is mainly described in the Jiang's paper ${ }^{[15]}$ based on the simulation of high-elevation air intake conditions under the condition of plug-in air intake distortion. The total pressure distribution of inlet and outlet are shown in Figure.3. Figure.4 shows the result of total pressure distribution of distortion under angle of attack. The results show that in the upper half of the flow field, due to the mixing effect, the distribution of the high and low pressure areas of the total pressure distribution are basically uniform. The high pressure area appears as a central circle with the largest area, and the low pressure area accounts for a very small area.

After entering the lower half of the flow field, due to the effect of the elbow structure, the airflow separation occurs, the growth rate of the low pressure area is accelerated. As the axial 
coordinate goes deeper into the curved section, the airflow separation is increasing. The lowpressure flow field at the rear of the bend continues to expand, and the low-energy flow spreads over a large area. The area of the high and low pressure zone decreases with the airflow, and the transition area between the high-pressure zone and the low-pressure zone gradually increases. Until the end of the inlet, the airflow separation phenomenon is gradually weakened due to the mixing of airflow, but the distribution of the total pressure in the outlet section is still uneven, and the low-pressure distribution of the total pressure inside the flow field is diffused. Compared with the uniform intake conditions, the uneven flow field in the lower half of the intake port under the angle of attack condition is alleviated. This is because the uneven intake environment reduces the air flow separation caused by the elbow structure to a certain extent.

After calculation, the distortion index of the intake port exit is $5.0 \%$. Compared with the uniform intake conditions, the total pressure distortion has all decreased. There is the reason to believe that the special structure of the S-shaped inlet will have better intake performance in a favourable direction than the bump inlet and sstraight inlet under uneven intake environments, such as when a fighter is manoeuvring through a stall.

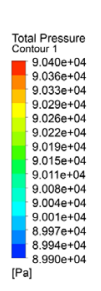

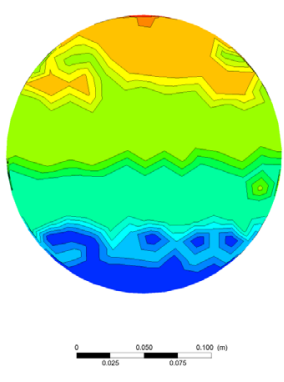

(a)Inlet
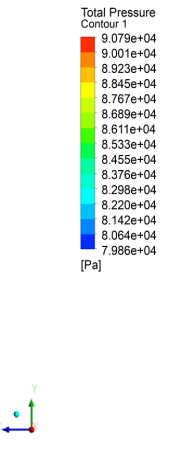

(b)Outlet
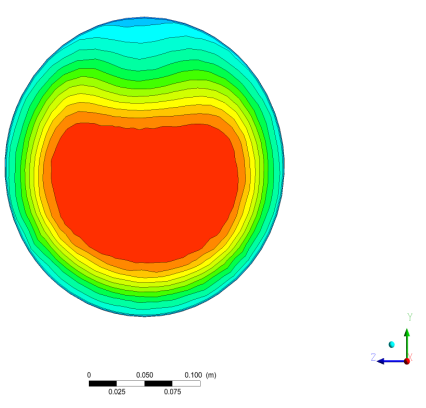

Fig. 3. Inlet and outlet total pressure distribution.

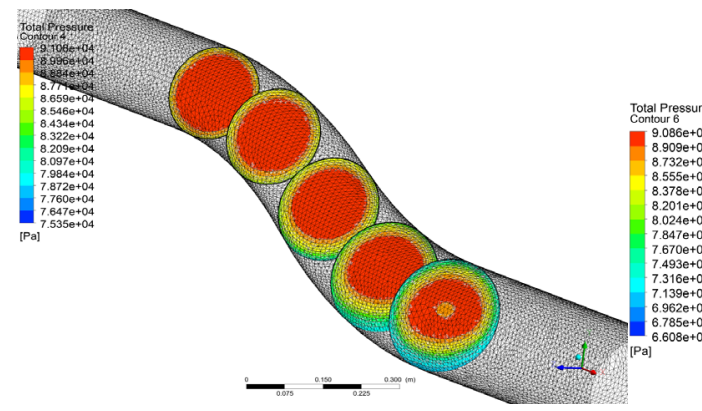

(a)Different faces

(b)Axial direction

Fig. 4. Total pressure distribution under angle of attack.

\subsection{Inlet distortion under indraft ground vortex}

This section simulates the distortion state of the flow field inside the intake port when the intake port is close to the ground and ground vortices which the low-energy vortex air are generated. The pressure at inlet is $100402 \mathrm{~Pa}$. Refer to the description in the relevant literature for the total pressure distribution of the intake section. The total pressure distribution at the inlet and outlet are shown in Figure 5. Figure. 6 shows the result of total pressure distribution 
under inlet distortion of sucking ground vortex. Results show that starting from the inlet section, as the flow field develops along the axial direction, the airflow separation brought by the boundary layer has limited influence, and the ground vortexes are sucked into the front end of the inlet and mixed with the high pressure region. As a whole, they are elongated in the pipeline as a low pressure area, and gradually close to the bottom of the wall during the development process. In the process of entering the bending section of the pipeline, it can be seen that the gradient of the elbow obviously magnifies the influence of the low-energy flow at the inlet. The boundary layer gradually accumulates to form a pressure difference with the main flow and secondary flow occurs at the bending position. The high pressure zone become as crescent shape because of the effect of second flow. Affected by the separation of airflow in the curved section, the range of the low-pressure zone continues to expand, and the highpressure zone entering the lower half of the flow field is squeezed and deformed, transitioning from a crescent shape to an arch shaped, and the edge of the high-pressure zone is gradually smooth. The high-energy core flow is further mixed with the low-energy flow, the area of the transition zone is reduced, and the pressure contours between the high and low pressure basins are more densely distributed.

However, due to the secondary flow and the separation of the air flow, more low-energy fluids accumulate at the bottom of the flow tube, and the average total pressure in the lowpressure zone shows a downward trend. At the end of the bend, the low-pressure area has obviously developed into a relatively large area. Even when the air flow separation at the end of the inlet is weakened and the air mixing effect is obvious, the high-pressure area is still shrinking slowly. It also shows the prospect of contraction at the low-pressure area. Compared with the analysis under uniform intake conditions, when the engine inlet sucks in the low-energy vortex, the unevenness of the total pressure from the inlet of the intake port to the curved section is more obvious, the boundary layer formed by the curved section is thicker, and the low energy flow caused by the fluid viscosity is relatively high. The phenomenon of air flow separation is more serious because of the secondary flow, which also leads to the distortion of the flow field in the second half of the intake duct obviously greater than the uniform intake conditions. After calculation, in this state, the distortion index of the intake port outlet is $8.2 \%$. Compared with the uniform intake conditions, the total pressure distortion index has increased significantly, indicating that under normal conditions, the inlet performance of S-shaped intake port will be weakened under the ground vortex be drawn into the inlet.
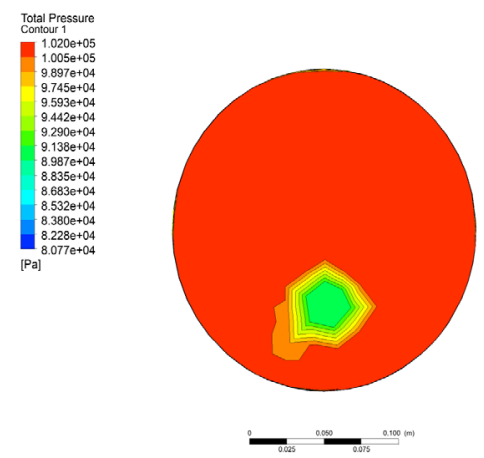

(a)Inlet

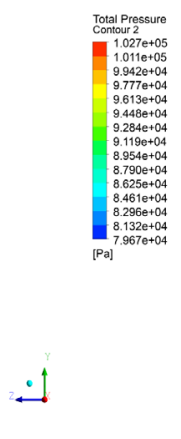

(b)Outlet

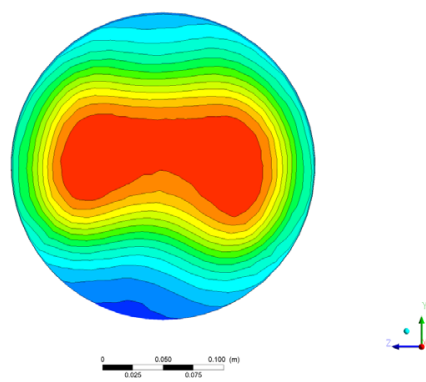

Fig. 5. Inlet and outlet total pressure distribution. 


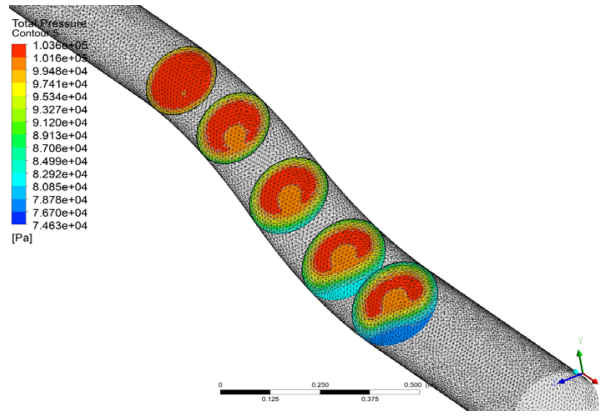

(a)Different faces

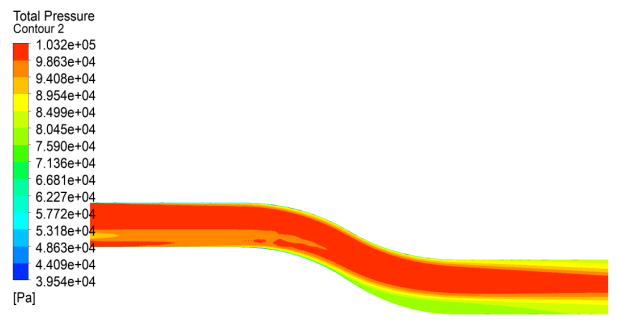

(b)Axial direction

Fig. 6. Total pressure distribution under angle of attack.

Table.1 shows the distortion index under different inlet conditions. Results show that distortion index may decrease or increase, it depends on the intake distortion conditions.

Table 1. Total pressure distortion index under different inflow condition.

\begin{tabular}{cc}
\hline Air inflow condition & Total pressure distortion index \\
\hline Uniform intake conditions & $7.5 \%$ \\
Angle of attack flight & $5.0 \%$ \\
Indraft ground vortex & $8.2 \%$ \\
\hline
\end{tabular}

\section{Conclusion}

According to the analysis of the results of the numerical simulation calculation, it can be known that the internal flow of S-shaped inlet is different because of the different mode of total pressure distortion. This paper numerical the inlet distortion under uniform intake conditions, angle of attack intake conditions, and ground vortex intake conditions, and analysed the distortion index under different conditions, the conclusions shown as following:

(1) Due to the curved shape of the S-shaped inlet, the separation phenomenon will occur even under uniform air intake conditions which makes effect on the subsequent flow field. In the absence of compressor suction, the total pressure distortion of the flow field is relatively high.

(2) Under certain flying conditions at an angle of attack, although the intake conditions are uneven, the airflow separation phenomenon of the S-shaped intake port is weakened, and the total pressure distortion is reduced, indicating that for some post-stall manoeuvres, the performance of the S-shaped intake port may be better than commonly used inlet ports.

(3) Inhalation of the ground vortex has a strong interference with the change of the flow field in the S-shaped inlet. A low-pressure region appears upstream of the flow field, and the boundary layer is thicker. The low-pressure region of the bend section expands faster and wider, and the degree of distortion at the outlet increases significantly, the distortion index will magnify, and the engine is close to the surge boundary.

The work is supported by Natural Science Basic Research Plan in Shaanxi Province of China. (Program No.2020JQ-477) 


\section{References}

1. XU Zhulin, GAO Rongzhao, DA Xingya. Assessment and measurement of total pressure distortion based on five-hole-probe for S-shaped inlet[J]. Journal of experiments in fluid mechanics, 2018, 32(04): 78-86

2. Zhang Zhen, Liu Dan, Huang Hao. Experimental investigation on flow control in an Sshaped inlet[J]. Chinese journal of applied mechanics, 2014, 31(02): 201-205

3. Briley W R \& Mcdonald H. Analysis and computation of viscous subsonic primary and second Flows. AIAA Journal,79:1453.

4. Levy R, Briley W R, Mcdonald H . Viscous/Secondary flow analysis for use with nonorthogonal coordinate systems. AIAA 83-0556.

5. Briley W R \& Mcdonald H. Three-dimensional viscous lows with large secondary velocity[J]. Fluid Mesh. 1984, 144: 47-77.

6. Weske J R. Pressure Loss in Ducts with Compound Elbows[C]. USA: NACA Wartime Rept. W-39, National Advisory Committee for Aeronautics Ohio, 1943.

7. Guo R, Seddon J. An Investigation of Swirl in an S-Duct[J]. The Aeronautical Quarterly, 1982, 33(1): 25-58.

8. Xiao Q, Tsai M H. Computation Transonic Diffuser Flows by a Lagged Turbulence Model [J]. Journal of Propulsion and Power, 2003, 19(3): 473-483.

9. Saha K, Singh S N, Seshadri V. Computational Analysis on Flow Through Transition SDiffusers: Effect of Inlet Shape[J]. Journal of Aircraft, 2007, 44(1): 187-193.

10. Gerolymos G A, Joly S, Mallet M, et al. Reynolds-Stress Model Flow Prediction in Aircraft-Engine Intake Double-S-Shaped Duct[J]. Journal of Aircraft, 2015, 47(4): 1368-1381.

11. Zhang Wei Li, Doyle D. Knight, Don Smith. Automated Design of a Three-Dimensional Subsonic Diffuser[J]. Journal of Propulsion \& Power, 2000, 16(6): 1132-1140.

12. Lin Qi, Guo Rongwei. Flow characteristics in an S-shaped rectangular-round diffuser at high incidence[J]. Acta aerodynamic sinica, 1989,7(2): 220-225.

13. Lin Qi, Guo Rongwei. The unsteady flow characteristics of an S-shaped inlet at high incidence[J]. Journal of Nanjing aeronautical institute, 1992, 24(1): 36-44.

14. Weng Xiaochai, Guo Rongwei. Investigation off pneumatic characteristics for a ventral S-shaped inlet at high incidence and low speed[J]. Journal of aerospace power, 2008, 23(9): 1573-1578.

15. Jiang Yong, Chen Ge, Kong Weidong, et al. An aero-engine response to the insertedboard air flow inlet distortion[J]. Journal of air force engineering university (Natural Science Edition), 2011, 12(03): 1-5. 